\title{
Análise das principais queixas ginecológicas no ambulatório escola da Universidade de Franca e correlação com dados epidemiológicos
}

\author{
Analysis of the main gynecological complaints in the outpatient school of the University of \\ Franca and correlation with epidemiological data \\ Análisis de las principales quejas ginecológicas en el ambulatorio escuela de la \\ Universidad de Franca y correlación con datos epidemiológicos
}

Karine Angélica Cintra ${ }^{1 *}$, Laura Ferreira de Almeida França ${ }^{1}$, Marcela Scalia ${ }^{1}$, Gabriela Magno Costa Ferreira ${ }^{1}$.

\section{RESUMO}

Objetivo: Analisar as principais queixas ginecológicas das pacientes do ambulatório escola de ginecologia, abordando também, dados sociodemográficos, exames complementares e as terapêuticas empregadas, além de correlacionar as queixas com os dados epidemiológicos. Metodologia: Estudo analítico e retrospectivo, através da análise de prontuários eletrônicos das pacientes do ambulatório escola da Universidade de Franca no período de novembro de 2017 a abril de 2018. Resultados: O tamanho amostral foi de 130 pacientes com idade média de 43 anos, sendo a maioria casadas, multíparas e sem atividade profissional. O motivo principal de procura do atendimento foi a realização de exame de rotina (37\%), seguida de queixas vaginais $(21,5 \%)$, alterações do ciclo menstrual $(18,5 \%)$ e dor pélvica (10\%). Em relação à conduta diagnóstica, em $87 \%$ dos casos foram solicitados exames complementares, como exames laboratoriais (57,5\%), colpocitogia oncótica de colo uterino $(56,5 \%)$, ultrassom transvaginal $(56,5 \%)$, mamografia $(46 \%)$ e outros. Houve associação significativa entre faixa etária e queixas e entre conduta terapêutica e queixas. Conclusão: Conclui-se que as principais queixas ginecológicas foram a realização de exames preventivos, seguida de queixas vaginais e alteração do ciclo menstrual. Houve associações relevantes entre as faixas etárias e queixas e entre conduta terapêutica e queixas.

Palavras-chave: Sinais e sintomas, Assistência Ambulatorial, Ginecologia.

\begin{abstract}
Objective: To analyze the main gynecological complaints of the patients of the gynecology outpatient clinic, also addressing socio-demographic data, complementary examinations and the therapies employed, besides correlating the complaints with the epidemiological data. Methodology: An analytical and retrospective study, through the electronic medical records analysis of patients from the University of Franca's outpatient clinic from November 2017 to April 2018. Results: The sample size was 130 patients with a mean age of 43 years. most married, multiparous and without professional activity. The main reason for seeking care was routine examination (37\%), followed by vaginal complaints $(21.5 \%)$, changes in the menstrual cycle $(18.5 \%)$ and pelvic pain (10\%). Regarding the diagnostic procedure, in $87 \%$ of the cases, complementary examinations were requested, such as laboratory tests $(57.5 \%)$, cervical oncotic colpocytogenesis $(56.5 \%)$, transvaginal ultrasound (56.5\%), mammography \%) and others. There was a significant association between age and complaints and between treatment and complaints. Conclusion: It was concluded that the main gynecological complaints were the preventive examinations, followed by vaginal complaints and alteration of the menstrual cycle. There were relevant associations between age groups and complaints and between therapeutic behavior and complaints.
\end{abstract}

Keywords: Signs and symptoms, Ambulatory Care, Gynecology.

${ }^{1}$ Universidade de Franca (UNIFRAN), Franca - SP. *E-mail: karinecintra@yahoo.com.br

SUBMETIDO EM: 1/2019

ACEITO EM: 2/2019

PUBLICADO EM: 4/2019

REAS/EJCH | Vol. 11 (9) | e368 | DOI: https://doi.org/10.25248/reas.e368.2019 Página 1 de 8 


\section{RESUMEN}

Objetivo: Analizar las principales quejas ginecológicas de las pacientes del ambulatorio escuela de ginecología, abordando también, datos sóciodemográficos, exámenes complementarios y las terapias empleadas, además de correlacionar las quejas con los datos epidemiológicos. Metodología: Estudio analítico y retrospectivo, a través del análisis de prontuarios electrónicos de las pacientes del ambulatorio escuela de la Universidad de Franca en el período de noviembre de 2017 a abril de 2018. Resultados: El tamaño muestral fue de 130 pacientes con edad media de 43 años, siendo la mayoría casadas, multíparas y sin actividad profesional. El motivo principal de búsqueda de la atención fue la realización de examen de rutina (37\%), seguida de quejas vaginales $(21,5 \%)$, alteraciones del ciclo menstrual $(18,5 \%)$ y dolor pélvico $(10 \%)$. En cuanto a la conducta diagnóstica, en el $87 \%$ de los casos se solicitaron exámenes complementarios, como exámenes de laboratorio $(57,5 \%)$, colpocitogia oncótica de cuello uterino $(56,5 \%)$, ultrasonido transvaginal (56,5\%), mamografía (46) \%) y otra. Se observó una asociación significativa entre el grupo de edad y las quejas y entre la conducta terapéutica y las quejas. Conclusión: Se concluye que las principales quejas ginecológicas fueron la realización de exámenes preventivos, seguida de quejas vaginales y alteración del ciclo menstrual. Hubo asociaciones relevantes entre las edades y las quejas y entre la conducta terapéutica y las quejas.

Palabras clave: Signos y síntomas, Atencíon Ambulatoria, Ginecología.

\section{INTRODUÇÃO}

A Ginecologia exerce sua prática dentro dos conhecimentos e experiências vivenciadas durante toda a vida da mulher, ou seja, desde a menacme até a pós-menopausa. Ela abrange os aspectos biológicos, mas também sociais, culturais e outros. É importante a conexão do corpo, mente, emoções, fatores sociais e ambientais para a compreensão do verdadeiro sentido das queixas trazidas até o consultório (PINHO L, et al., 2014).

Durante o ensino acadêmico e principalmente ao colocarem em prática o conteúdo adquirido ao longo dos anos, os estudantes de medicina percebem que a responsabilidade profissional constitui uma barreira importante de expressão no contato com a paciente. Muitas vezes, a teoria e a prática são insuficientes para atender às demandas subjetivas que surgem na interação que se estabelece em uma consulta ginecológica. Para fazer o diagnóstico e adequar o tratamento, não basta o conhecimento técnico, é necessário considerar que cada atendimento tem suas particularidades (CORDEIRO NA, et al., 2010).

Desta forma, a atuação de estudantes de medicina nessa área é de fundamental importância para a formação global na atenção à saúde da mulher. Algumas dificuldades na especialidade devem ser contornadas, como, por exemplo, o constrangimento inicial das pacientes pela realização dos exames específicos pelos estudantes, o próprio receio dos graduandos em abordar determinados assuntos mais delicados, como a sexualidade, por exemplo, e o impacto que o sexo do acadêmico pode causar (RIO SMP, et al., 2013; BARATA LRB, et al., 2010).

Assim, deve-se considerar a importância dos profissionais de saúde se aproximarem da experiência de vida de cada pessoa atendida com objetivo de melhor acolher suas necessidades. Dessa forma, os ambulatórios didático-assistenciais de ginecologia favorecem a formação de profissionais aptos a enfrentarem os problemas de saúde da população feminina (SOUZA IP, et al., 2016; PINHO L, et al., 2014; BARATA LRB, et al., 2010).

$\mathrm{Na}$ anamnese ginecológica, a queixa principal é transcrita ao prontuário de modo fiel às expressões usadas pela paciente. Em muitos casos, o motivo da consulta ginecológica ocorre para prevenção oncológica, orientação anticoncepcional, pré-natal e não apenas por patologias (DRUSZCZ RMB, et al., 2014).

Para Martinez JB (2013), a existência de qualquer sintoma referido pela paciente deve ser registrado e investigado, pesquisando dados que possam estar com ele relacionados, detalhando-os na história da moléstia atual, para, posteriormente, poderem ser sanados com a conduta específica. 
Diante disso, o conhecimento das principais queixas ginecológicas encontradas no serviço de ginecologia geral favorece o planejamento docente e discente sobre as atividades a serem desenvolvidas no decorrer do período letivo em prol da população atendida. Portanto, o objetivo geral do estudo foi avaliar as queixas ginecológicas mais prevalentes e correlacioná-las ao perfil epidemiológico desse público.

\section{METODOLOGIA}

Trata-se de um estudo analítico, observacional e retrospectivo. O estudo foi realizado no Ambulatório Escola da Universidade de Franca (UNIFRAN) localizado em Franca, no Estado de São Paulo.

Foram incluídas 130 pacientes que procuraram o atendimento do Ambulatório Escola de novembro de 2017 a abril de 2018 e excluídas as pacientes que não compareceram as consultas marcadas no mesmo período. Avaliou-se as queixas principais registradas, assim como os dados epidemiológicos das pacientes. A análise dos prontuários foi realizada por três acadêmicas do curso de Medicina da Universidade de Franca.

Ressalta-se que o trabalho em questão não possui Termo de Ciência Livre e Esclarecido por se tratar de análise retrospectiva, através do estudo de prontuários médicos, mantendo sigilo sobre a identificação das pacientes, sem geração de danos. Buscando zelar pela integridade ética das envolvidas na pesquisa o projeto foi submetido à análise e aprovação pelo Comitê de Ética em Pesquisa da Fundação Santa Casa de Misericórdia de Franca, com CAAE de número 80728717.5.0000.5438, e seguiu as diretrizes e normas regulamentadoras de pesquisa envolvendo seres humanos, estabelecidas pela resolução 466/2012.

Os dados coletados nos prontuários analisados foram registrados em planilhas confeccionadas com 0 software Microsoft $\Theta^{\circledR}$ Office Excel $\AA_{2010}$, onde foi construído um banco de dados. A análise foi realizada através de estatísticas descritivas utilizando medidas de frequência, por meio do método qui-quadrado. $O$ nível de significância adotado foi de $p<0.5$.

\section{RESULTADOS}

A amostra estudada foi composta de 130 pacientes, com média de idade de 43 anos. Com relação aos dados epidemiológicos, as pacientes foram questionadas sobre estado civil, paridade e profissão. Ao avaliar o estado civil das pacientes, dividiu-se a descrição em casada/união estável, solteira, desquitada/divorciada e viúva. Para a análise da paridade, as classificações utilizadas foram: nulípara, primípara, multípara e não informado (Tabela 1).

Tabela 1 - Caracterização dos dados epidemiológicos. Franca - SP, 2018.

\begin{tabular}{lcc} 
Caracterização & Quantidade & Percentual \\
\hline Idade Média \pm d. Padrão: 43,03 $\pm 14,43$ anos & 130 & $100 \%$ \\
Estado Civil & 78 & $60 \%$ \\
$\quad$ Casada / União Estável & 35 & $27 \%$ \\
$\quad$ Solteira & 9 & $7 \%$ \\
$\quad$ Desquitada / Divorciada & 8 & $6 \%$ \\
$\quad$ Viúva & & \\
Paridade & 80 & $61,5 \%$ \\
$\quad$ Nulípara & 20 & $15,5 \%$ \\
$\quad$ Primípara & 28 & $21,5 \%$ \\
$\quad$ Multípara & 2 & $1,5 \%$ \\
Não informado & & \\
Profissão & 47 & $36 \%$ \\
Não trabalha & 78 & $60 \%$ \\
Trabalha & 5 & $4 \%$ \\
Não informado & & \\
\hline
\end{tabular}

Fonte: Dados da pesquisa, 2018. 
Em relação às queixas principais, observou-se que 37\% tratava-se de rotina ginecológica, como coleta de citologia oncótica e rastreamento mamográfico, seguida por queixas vaginais $(21,5 \%)$, alterações do ciclo menstrual (18,5\%), como aumento ou diminuição do fluxo menstrual, e dor pélvica (10\%). Dentre as queixas vaginais, $46,5 \%$ o motivo da consulta era leucorréia, $28,5 \%$ sangramento vaginal, $14 \%$ lesão vulvovaginal e $11 \%$ prurido. Em queixas mamárias, foram agrupados os nódulos mamários (33,5\%), mastalgia (55\%) e 0 aumento do volume mamário (11\%) e em outras queixas foram enquadradas a incontinência urinária e/ou urgência miccional, totalizando $5,5 \%$ (Gráfico 1).

Gráfico 1 - Caracterização das queixas principais. Franca - SP, 2018.

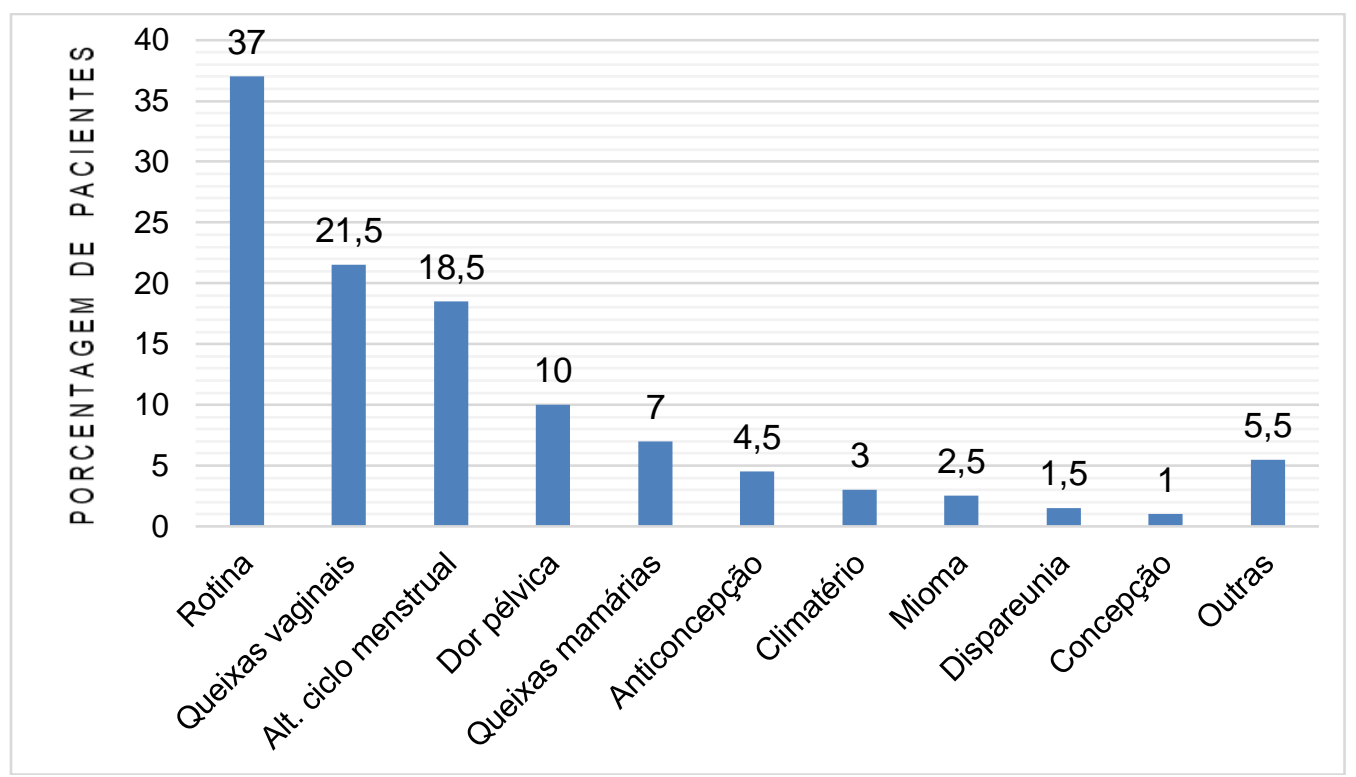

Fonte: Dados da pesquisa, 2018.

Sobre a propedêutica realizada em cada consulta médica, $87 \%$ das pacientes tiveram exames complementares solicitados e em $13 \%$ não consta exames. Dentre os exames, $57,5 \%$ foram exames laboratoriais, $56,5 \%$ ultrassom transvaginal, $56,5 \%$ de citologia oncótica, $46 \%$ mamografia, $11,5 \%$ ultrassom de mamas, $10 \%$ densitometria óssea e $13 \%$ outros (Gráfico 2 ).

Gráfico 2 - Caracterização das condutas diagnósticas. Franca-SP, 2018.

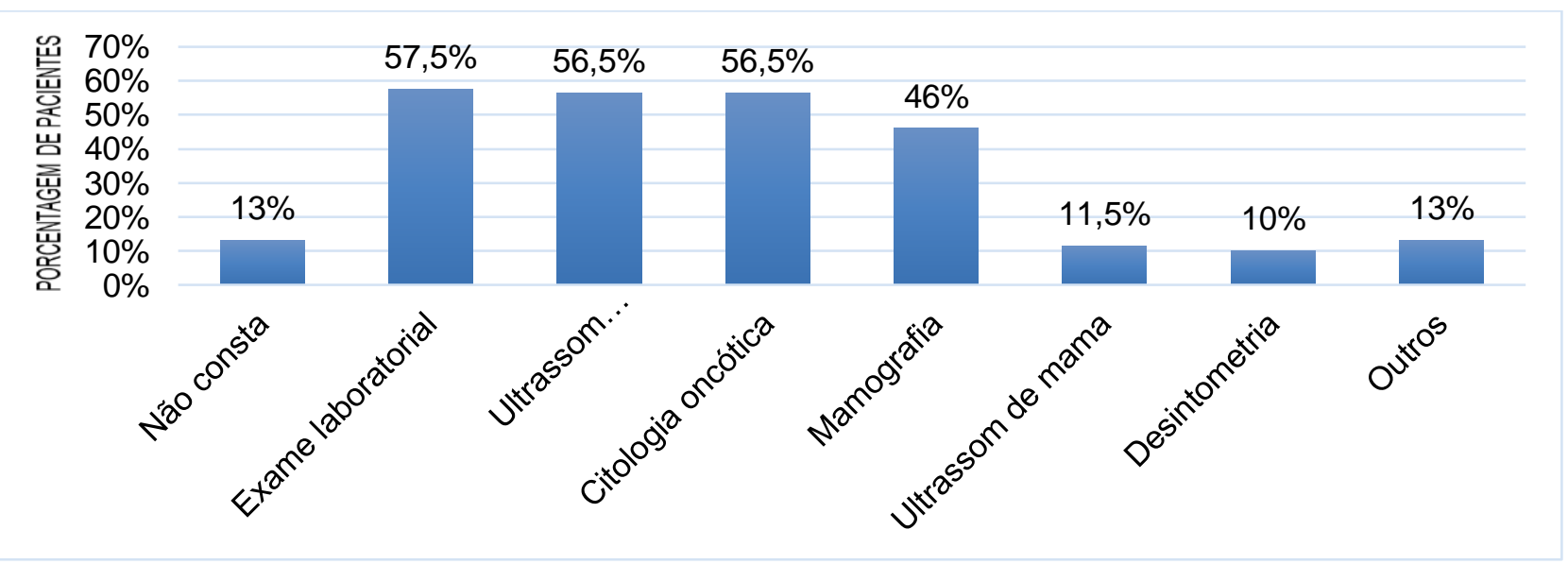

Fonte: Dados da pesquisa, 2018. 
Também foi avaliada a relação da queixa principal com alguns dados sociodemográficos. Houve associação significativa $\left(x^{2}=30,26 ; p=0,0110^{*}\right)$ entre as queixas ginecológicas e as faixas etárias, mostrando que as demandas por atendimento não se distribuem de forma casual entre as diferentes faixas etárias.

Diante dessa relação, obteve-se que nas pacientes com idade menor ou igual a 18 anos houve maior frequência de queixas relacionadas à alteração do ciclo menstrual. Por outro lado, nas pacientes entre $19 \mathrm{e}$ 40 anos e nas de 41 a 49 anos, existiu maior incidência de exames de rotina como solicitação. Por fim, entre as pacientes com idade maior que 50 anos, houve maior frequência de queixas vaginais (Gráfico 3).

Gráfico 3 - Faixas etárias associadas às principais queixas. Franca-SP, 2018

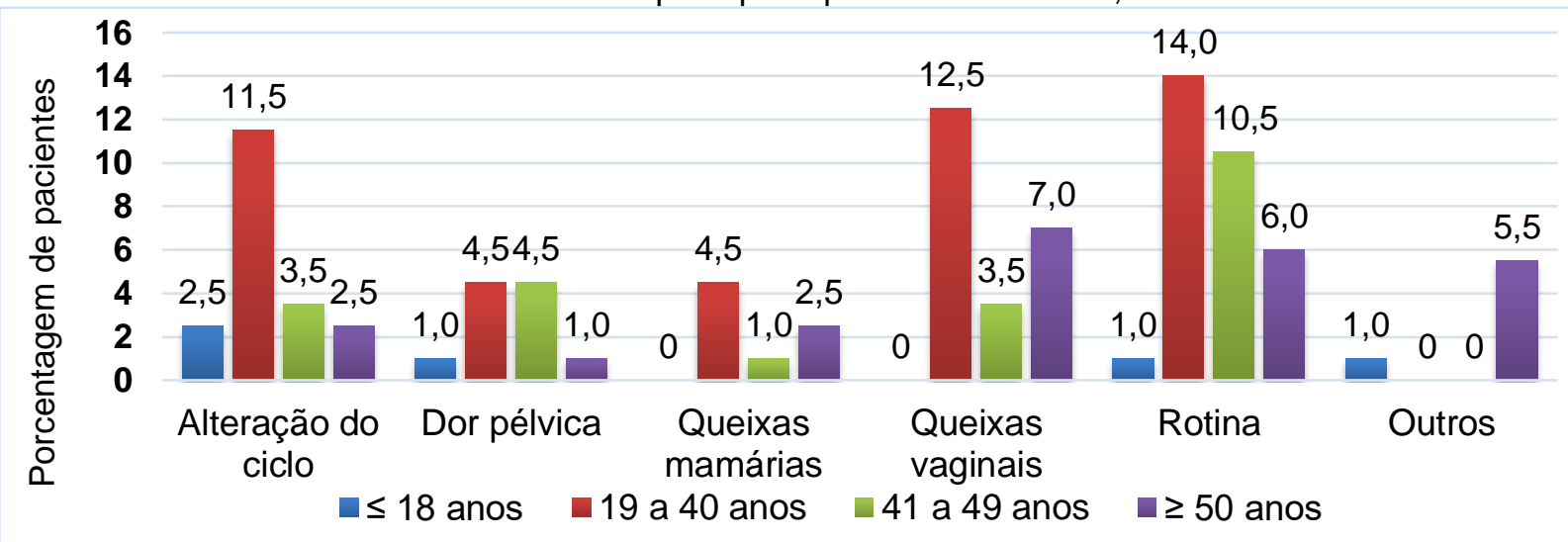

Fonte: Dados da pesquisa, 2018.

Quanto à relação da queixa atual e estado civil não foi observado associação significativa $\left(X^{2}=2,08\right.$; $p=0,5569)$. Entretanto, obteve-se associação significativa entre queixas principais e as condutas terapêuticas $\left(X^{2}=869,7 ; p=0,0001\right)$. Para a queixa de dor pélvica e de alteração do ciclo menstrual, a conduta tomada mais frequente foi a prescrição de anti-inflamatórios não esteroidais (AINES). Para queixas vaginais, a terapêutica mais utilizada foi o tratamento de vaginoses. Em relação ao exame de rotina e a solicitação de anticoncepcionais, a conduta mais adotada foi a prescrição dos mesmos. E por último, para as queixas mamárias, houve equivalência de 3 abordagens: prescrição de AINES, tratamento de atrofia vaginal e outros (Gráfico 4).

Gráfico 4 - Prescrições associadas as principais queixas. Franca-SP, 2018.

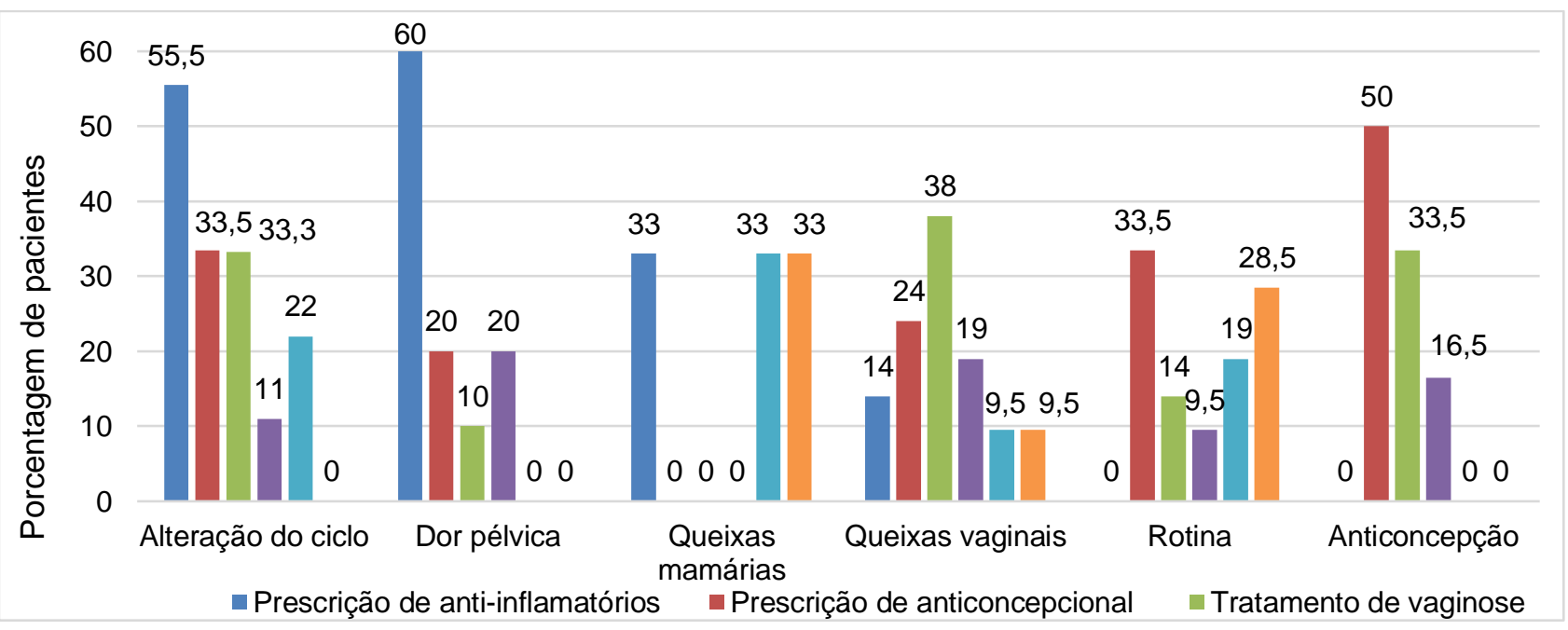

Fonte: Dados da pesquisa, 2018. 


\section{DISCUSSÃO}

A consulta ginecológica é parte essencial da vida da mulher, já que abrange a prevenção, diagnóstico e tratamento de patologias do sistema reprodutor. Neste sentido, na dinâmica do atendimento à mulher, é necessário correlacionar as questões ginecológicas com o social e psicológico, abordando todo o contexto da vida da mulher. Desta forma, assistindo-a de maneira holística, humanizada e qualificada (CORDEIRO NA, et al., 2010).

Nesse contexto, durante as consultas ginecológicas das pacientes atendidas no ambulatório escola da UNIFRAN, avaliou-se as principais queixas ginecológicas trazidas, em que se observou a prevalência de três principais, as quais serão discutidas a seguir. Inicialmente, tem-se a solicitação dos principais exames de rastreio, como a citologia oncótica e a mamografia, como o maior motivo de procura por atendimento.

Quanto a citologia oncótica, ressalta-se que apareceu como segundo exame complementar mais solicitado. Conforme o Ministério da Saúde (Brasil, 2013), essa alta prevalência pode ser justificada pelo fato desse exame ser preconizado na população feminina com vida sexual ativa, e como prática de rastreamento periódico em mulheres de 25-64 anos.

No que diz respeito à mamografia, o terceiro exame complementar mais solicitado nas consultas ginecológicas, o Ministério da Saúde (Brasil, 2016) salienta que o Brasil ainda apresenta falhas na abordagem do rastreamento do câncer mamário, cujo diagnóstico e tratamento frequentemente não são realizados em tempo oportuno, gerando menor sobrevida das pessoas diagnosticadas, em comparação com países desenvolvidos.

Em relação às queixas vaginais, segunda colocada em prevalência, incluiu-se o corrimento vaginal como subgrupo, considerado uma síndrome comum, que ocorre principalmente na idade reprodutiva, segundo o Ministério da Saúde (Brasil, 2015), e tem como etiologia causas infecciosas e não infecciosas, por isso, a história clínica deverá ser minuciosa, com informações sobre comportamentos e práticas sexuais, data da última menstruação, práticas de higiene vaginal e uso de medicamentos tópicos ou sistêmicos.

Ainda dentro de queixas vaginais, o prurido vulvar, encontrada nos relatos de $11 \%$ das mulheres pesquisadas, tem-se que é um sintoma comum entre as mulheres. Um número superior a $10 \%$ das mulheres procura assistência médica motivadas por esse quadro (LUCAS ICRN, et al., 2012). A vulva é sede de várias doenças locais ou de manifestações de diversas patologias sistêmicas e frequentemente tem como sintoma o prurido e/ou a dor. Estes sintomas podem estar associados a processos inflamatórios, imunológicos, distúrbios metabólicos e neoplasias (OLIVEIRA R, et al., 2010).

No que concerne às lesões vulvovaginais (14\%), também prevalentes, de acordo com o Ministério da Saúde (Brasil, 2015), deve-se considerar que embora a úlcera genital esteja frequentemente associada às infecções sexualmente transmissíveis (IST) na população sexualmente ativa, a queixa não é exclusividade das IST e pode estar associada com infecções inespecíficas por fungos, vírus ou bactérias, como em dermatoses bolhosas, líquen plano erosivo, lesões traumáticas e até mesmo lesões malignas.

Sobre a terceira queixa mais prevalente, alterações do ciclo menstrual (18,5\%), destaca-se o sangramento uterino anormal (SUA), que possui etiologia multifatorial e pode ser dividida em causas orgânicas ou disfuncionais. Dentre os fatores orgânicos estão incluídas situações correlatas da gravidez, doenças sistêmicas ou pélvicas, miomas, traumas e uso de medicamentos. Já os fatores disfuncionais são alterações nos mecanismos endócrinos que controlam a menstruação, ocorrido na ausência de gestação. (SANTOS EKA, et al., 2012).

No que diz respeito a correlação realizada de queixas principais e faixa etária, observou-se que um estudo descritivo, realizado em uma escola do município de São Paulo, com número de 40 estudantes de 15 a 19 anos, teve maior frequência de avaliação de rotina (30\%) nas procuras por atendimento, seguido por anticoncepção $(27,5 \%)$, de acordo com Ramos ACP et al. (2014), fato que não coincide com o resultado encontrado no presente estudo. Supõe-se que essa diferença se dá pelo fato de o estudo em comparação ter um número reduzido de participantes, de idade mais restrita. 
Com relação aos motivos de consulta ginecológica mais frequentes entre as adolescentes encontram-se a avaliação de rotina do desenvolvimento da puberdade, problemas relacionados aos ciclos menstruais, cólicas, corrimento e o desejo de contracepção, segundo Rehme MFB e Cabral Z (2017). No estudo presente, tem-se que as queixas principais mais incidentes entre as pacientes com idade menor ou igual a 18 anos, foram a alteração do ciclo menstrual, seguida por avaliação de rotina e dor pélvica, fato condizente com a literatura.

No trabalho vigente, verificou-se como queixa mais frequente em mulheres de 41 a 49 anos a solicitação de exames de rotina e em mulheres com idade maior ou igual a 50 anos as alterações vaginais. Em estudo realizado por Malheiros ESA et al. (2014) na cidade de São Luís (MA) com 1.210 entrevistadas de idade média de 48 anos constatou-se a predominância dos sintomas vasomotores (77,8\%). Sugere-se que essa diferença tem como motivo o fato de a síndrome do climatério apresentar um quadro clínico altamente variável, posto que, é altamente influenciada por fatores ligados ao meio e a peculiaridade de cada mulher.

Quanto a relação entre a queixa atual e estado civil das pacientes, percebeu-se que não houve associação significativa. Assim, considerou-se a possibilidade de que as queixas ginecológicas tenham mais associação com o comportamento sexual do que com o estado civil. Conforme Zimmermmann JB, et al. (2011), o número de parceiros, o uso de contraceptivos e a idade precoce de iniciação sexual seriam os fatores que geram maior vulnerabilidades em relação as queixas vaginais, incluindo as Doenças Sexualmente Transmissíveis.

Em se tratando da relação entre as queixas principais e as condutas terapêuticas, constatou-se que para as queixas vaginais, a terapêutica mais utilizada foi o tratamento de vaginoses, provavelmente pelo fato de as queixas de corrimento vaginal terem sido as mais frequentes dentro desse grupo. O Ministério da Saúde (Brasil, 2015) sugere que o tratamento de leucorreia seja norteado pela microscopia, se disponível.

Por fim, em referência as alterações do ciclo menstrual, Pinto et al. (2017) refere que o tratamento do SUA depende se as causas são estruturais ou não. Em relação as causas estruturais, deve-se considerar que as alterações neoplásicas e pré-neoplásicas do endométrio têm grande importância como diagnóstico diferencial. Já o SUA de causa não estrutural, a conduta terapêutica baseia-se na ação dos hormônios e de outros mediadores inflamatórios sobre o endométrio, além do controle hemostático do sangramento, conforme visto no trabalho atual, que teve como principal conduta terapêutica a prescrição de AINES. Isto sugere, então, que dentre as alterações do ciclo menstrual analisadas predominaram as de origem funcional.

\section{CONCLUSÃO}

O estudo demonstrou que as principais queixas ginecológicas das pacientes que procuraram o ambulatório escola de ginecologia foram a realização de exames preventivos, seguida de queixas vaginais e alteração do ciclo menstrual. Houve associação significativamente relevantes entre as faixas etárias e queixas principais e entre condutas terapêuticas e queixas. A análise detalhada do perfil de queixas das pacientes que procuram consulta médica permite melhorar os protocolos de atendimento, possibilitando a identificação de demandas terapêuticas medicamentosas ou não, e, consequentemente, melhorando a assistência à população, além de contribuir para a construção de novos trabalhos científicos nessa área de conhecimento.

\section{REFERÊNCIAS}

1. BARATA LRB, et al. Hospitais de ensino e o sistema único de saúde. RAS, 2010; Vol. 12, № 46.

2. BRASIL. Ministério da Saúde. Secretaria de Atenção à Saúde. Departamento de Atenção Básica. Cadernos de Atenção Básica: Controle dos cânceres do colo do útero e da mama. Brasília: Ministério da Saúde; 2013.

3. BRASIL. Ministério da Saúde. Secretaria de Vigilância em Saúde. Departamento de DST, Aids e Hepatites Virais. Protocolo Clínico e Diretrizes Terapêuticas para Atenção Integral às Pessoas com Infecções Sexualmente. Brasília: Ministério da Saúde; 2015.

4. BRASIL. Ministério da Saúde. Protocolos da Atenção Básica: Saúde da Mulheres. Instituto Sírio-Libanês de Ensino e Pesquisa. Brasília: Ministério da Saúde; 2016. 
5. CORDEIRO NA, et al. Questões da clínica ginecológica que motivam a procura de educação médica complementar: um estudo qualitativo. Revista brasileira de educação médica, 2010; 34 (2): 255-260.

6. DRUSZCZ RMB, et al. Semiologia ginecológica: o atendimento da mulher na atenção primária à saúde. Arq Med Hosp Fac Cienc Med Santa Casa São Paulo, 2014; 59(3):144-151.

7. LUCAS ICRN, et al. Prurido vulvar: diagnóstico diferencial. Revista de Medicina e Saúde de Brasília, 2012; 2; $1(1): 20-5$.

8. MALHEIROS ESA, et al. Síndrome climatérica em uma cidade do Nordeste brasileiro: um inquérito domiciliar. Rev Bras Ginecol Obstet., 2014; 36(4):163-9.

9. MARTINEZ JB. Semiologia Médica Geral e Especializada. Rio de Janeiro: Guanabara Koogan, 2013.

10. OLIVEIRA R, et al. Conduta na dor e prurido vulvar. FEMINA, 2010; 38(15).

11. PINHO L, et al. Nascer e Crescer. Revista de Pediatria do Centro Hospitalar do Porto. Departamento de Ensino, Formação e Investigação, 2013; 22(4): 210-215.

12. PINTO CLB, et al. Sangramento Uterino Anormal. FEBRASGO, 2017; n. 7: 2525-6416

13. RAMOS ACP, et al. Consulta ginecológica sob a ótica de adolescentes. R. Enferm. Cent. O. Min, 2014; 3(4):12481261.

14. REHME MFB, CABRAL Z. Atendendo a adolescente no consultório. FEBRASGO, 2017; n. 4.

15. RIO SMP, et al. Vivência das mulheres atendidas por alunos de medicina em consulta ginecológica. Revista brasileira de educação médica, 2013; 37 (4): 492-500.

16. SANTOS EKA, et al. Atenção integral à saúde da mulher: enfermagem. Universidade Federal de Santa Catarina, $2012 ; 2$. ed.

17. SOUZA IP, et al. Adolescer e adoecer na perspectiva de jovem e família. Ciencia y enfermeria XXII (3), 2016.

18. ZIMMERMMANN JB, et al. Aspectos ginecológicos e obstétricos de pacientes atendidas nos serviços público e privado de saúde. Há diferenças? Rev Bras Ginecol Obstet, 2011; 33(12):401-7. 